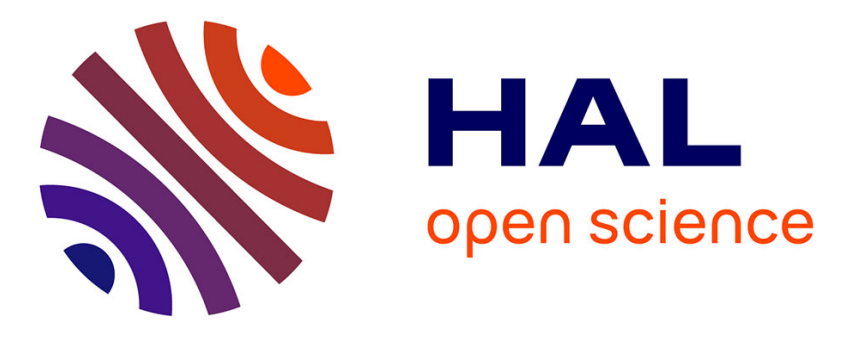

\title{
On Dynamical Complexity of Surjective Ultimately Right-Expansive Cellular Automata
}

\author{
Joonatan Jalonen, Jarkko Kari
}

\section{To cite this version:}

Joonatan Jalonen, Jarkko Kari. On Dynamical Complexity of Surjective Ultimately Right-Expansive Cellular Automata. 24th International Workshop on Cellular Automata and Discrete Complex Systems (AUTOMATA), Jun 2018, Ghent, Belgium. pp.57-71, 10.1007/978-3-319-92675-9_5 . hal01824870

\section{HAL Id: hal-01824870 \\ https://hal.inria.fr/hal-01824870}

Submitted on 27 Jun 2018

HAL is a multi-disciplinary open access archive for the deposit and dissemination of scientific research documents, whether they are published or not. The documents may come from teaching and research institutions in France or abroad, or from public or private research centers.
L'archive ouverte pluridisciplinaire HAL, est destinée au dépôt et à la diffusion de documents scientifiques de niveau recherche, publiés ou non, émanant des établissements d'enseignement et de recherche français ou étrangers, des laboratoires publics ou privés. 


\title{
On Dynamical Complexity of Surjective Ultimately Right-Expansive Cellular Automata
}

\author{
Joonatan Jalonen ${ }^{\star}$ and Jarkko Kari ${ }^{\star \star}$ \\ University of Turku
}

\begin{abstract}
We prove that surjective ultimately right-expansive cellular automata over full shifts are chain-transitive. This immediately implies Boyle's result that expansive cellular automata are chain-transitive. This means that the chain-recurrence assumption can be dropped from Nasu's result that surjective ultimately right-expansive cellular automata with right-sided neighborhoods have the pseudo-orbit tracing property, which also implies that the (canonical) trace subshift is sofic. We also provide a theorem with a simple proof that comprises many known results including aforementioned result by Nasu. Lastly we show that there exists a right-expansive reversible cellular automaton that has a non-sofic trace and thus does not have the pseudo-orbit tracing property. In this paper we only consider cellular automata over full shifts, while both Nasu and Boyle obtain their results over more general shift spaces.
\end{abstract}

\section{Introduction}

Questions regarding what kind of a subshift an expansive cellular automaton is conjugate to has been considered at least in $[16,10,4,2,17,18]$. In [16] Nasu presented his textile systems and used them to show that positively expansive two-sided cellular automata are conjugate to full shifts. Around the same time, using more standard methods of symbolic dynamics, Kưrka [10] obtained independently that positively expansive cellular automata are conjugate to subshifts of finite type. Kurka explicitly stated this only for two-sided cellular automata but the proof also works for one-sided positively expansive cellular automata. Blanchard and Maass [4] improved the case of positively expansive one-sided cellular automata and showed that these are conjugate to full shifts. Next Nasu [17], again using textile systems, proved that one-sided expansive cellular automata are conjugate to subshifts of finite type. Later on Nasu [18] proved that also expansive two-sided cellular automata are conjugate to subshifts of finite type if the local neighborhood is one-sided. In this Nasu used Boyle's result from [1] that expansive cellular automata are chain-transitive. Using an additional assumption to guarantee chain-transitivity Nasu extended his result for two-sided surjective ultimately right-expansive cellular automata with right-sided neighborhoods. By ultimately right-expansive we mean that if we know with finite

\footnotetext{
* Research supported by the Finnish Cultural Foundation.

** Research supported by the Academy of Finland Grant 296018
} 
precision the entire history and future of a point, then we know the entire right side of the space-time diagram.

In this paper we restrict to cellular automata over full shifts. We show that a surjective ultimately right-expansive cellular automaton is automatically chaintransitive (Theorem 1). The proof is elementary. It then follows that one of Nasu's assumptions can be dropped, at least for cellular automata over full shifts. We will also provide a single theorem (Theorem 2), with a simple proof, that covers many of the cases mentioned in the previous paragraph (for full shifts). The proof is inspired by Taati's proof that a cellular automaton that is reversible over its limit set is stable, i.e. reaches the limit set in finite time [19].

Lastly we show that the requirement of right-expansivity alone is not enough: We give an example of a right-expansive cellular automaton over a full shift with a trace that is not sofic. This is directly based on the construction of the second author and Lukkarila [9]. In the case of cellular automata over non-transitive subshifts, the appendix of [1] contains an example by Fiebig of an expansive cellular automaton over a non-transitive subshift whose trace is not an SFT.

\section{Preliminaries}

Notations For two integers $i, j \in \mathbb{Z}$ such that $i<j$ the interval from $i$ to $j$ is denoted $[i, j]=\{i, i+1, \ldots, j\}$. We also denote $[i, j)=\{i, i+1, \ldots, j-1\}$ and $(i, j]=\{i+1, \ldots, j\}$. Notation $\mathbb{M}$ is used when it does not matter whether we use $\mathbb{N}$ or $\mathbb{Z}$. Composition of functions $f: X \rightarrow Y$ and $g: Y \rightarrow Z$ is written as $g f$ and defined by $g f(x)=g(f(x))$ for all $x \in X$.

Topological dynamics A (topological) dynamical system is a pair $(X, f)$ where $X$ is a compact metric space and $f$ a continuous map $X \rightarrow X$. Let $(X, f)$ and $(Y, g)$ be two dynamical systems. A continuous map $\phi: X \rightarrow Y$ is a homomorphism if $\phi f=g \phi$. If $\phi$ is surjective, it is a factor map, and $(Y, g)$ is a factor of $(X, f)$. Let $d: X \times X \rightarrow \mathbb{R}_{+} \cup\{0\}$ be the metric considered. A sequence $\left(x_{i}\right)_{i \in \mathbb{Z}}$ is an orbit of $f$ if $f\left(x_{i}\right)=x_{i+1}$ for every $i \in \mathbb{Z}$. Let $x, y \in X$. There is an $\varepsilon$-chain from $x$ to $y$ if there exists $n>0$ and a sequence $x=x_{0}, x_{1}, \ldots, x_{n}=y \in X$ such that $d\left(f\left(x_{i}\right), x_{i+1}\right)<\varepsilon$, for all $i \in\{0,1, \ldots, n-1\}$. Infinite $\varepsilon$-chains are called $\varepsilon$-pseudo-orbits. The dynamical system $(X, f)$

- is transitive if for all non-empty open sets $U, V$ there exists $n>0$ such that $F^{n}(U) \cap V \neq \emptyset$.

- is chain-recurrent if for all $x \in X$ and $\varepsilon>0$ there exists an $\varepsilon$-chain from $x$ to $x$.

- is chain-transitive if for all $x, y \in X$ and $\varepsilon>0$ there exists an $\varepsilon$-chain from $x$ to $y$.

- has pseudo-orbit tracing property, often also called the shadowing property, if for all $\varepsilon>0$ there exists $\delta>0$ such that for any $\delta$-pseudo-orbit $\left(x_{i}\right)_{i \in \mathbb{Z}}$ there exists an orbit $\left(y_{i}\right)_{i \in \mathbb{Z}}$ such that $d\left(x_{i}, y_{i}\right)<\epsilon$. 
Symbolic dynamics The set of infinite sequences over an alphabet $A$ indexed by $\mathbb{M}$ is $A^{\mathbb{M}}$. An element $c \in A^{\mathbb{M}}$ is a configuration. A configuration is a function $\mathbb{M} \rightarrow A$ and we denote $c(i)=c_{i}$ for $i \in \mathbb{M}$. Let $D \subseteq \mathbb{M}$ be finite and $u \in A^{D}$, then the set $[u]=\left\{c \in A^{\mathbb{M}} \mid c_{D}=u\right\}$ is called a cylinder. Let $A$ have the discrete topology and $A^{\mathbb{M}}$ the product topology. Cylinders form a countable clopen (open and closed) base of this topology. We consider $A^{\mathbb{M}}$ to be a metric space with the metric

$$
d(c, e)= \begin{cases}2^{-\min \left(\left\{|i| \mid c_{i} \neq e_{i}\right\}\right)}, & \text { if } c \neq e \\ 0, & \text { if } c=e\end{cases}
$$

for all $c, e \in A^{\mathbb{M}}$. It is well-known that this metric induces the same topology as defined above, and that this space is compact.

For any $n \in \mathbb{N} \backslash\{0\}$ we denote $A^{n}=A^{[0, n)}$, and $A^{+}=\bigcup_{n \in \mathbb{N} \backslash\{0\}} A^{n}$ and call any subset $L \subset A^{+}$a language. The language $L$ is finite if $L$ is finite, and regular if it is recognized by a finite state automaton. If $L_{1}, L_{2} \subseteq A^{+}$are two languages, then their concatenation is $L_{1} L_{2}=\left\{u v \in A^{+} \mid u \in L_{1}\right.$ and $\left.v \in L_{2}\right\}$.

The shift map $\sigma: A^{\mathbb{M}} \rightarrow A^{\mathbb{M}}$, defined by $\sigma(c)_{i}=c_{i+1}$ for all $i \in \mathbb{M}$, is continuous. The dynamical system $\left(A^{\mathbb{M}}, \sigma\right)$ is the full $(A$-) shift. A dynamical system $(X, \sigma)$, where $X \subseteq A^{\mathbb{M}}$ is non-empty, topologically closed, and $\sigma^{m}(X) \subseteq$ $X$ for all $m \in \mathbb{M}$, is a shift space or a subshift. When it does not cause confusion, we will simply talk about a subshift $X$. A configuration $c \in A^{\mathbb{M}}$ avoids $u \in A^{n}$ if $\sigma^{i}(c)_{[0, n)} \neq u$ for all $i \in \mathbb{M}$, otherwise $u$ appears in $c$. Let $S \subseteq A^{+}$, and let $X_{S}$ be the set of configurations that avoid $S$, i.e. $X_{S}=\left\{c \in A^{\mathbb{M}} \mid \forall u \in S: c\right.$ avoids $\left.u\right\}$. It is well-known that the given topological definition of subshifts is equivalent to saying that there exists a set of forbidden words $S$ such that $X=X_{S}$. If there exists a finite set $S$ such that $X=X_{S}$, then $X$ is a subshift of finite type (SFT). If $Y$ is a factor of an SFT, then it is a sofic shift. An equivalent characterization of sofic shifts is that the set of forbidden words is a regular language.

The language of a subshift $(X, \sigma)$ is the set of words that appear in some configuration of $X$, and is denoted by $\mathcal{L}(X)$. We also denote $\mathcal{L}_{n}(X)=\mathcal{L}(X) \cap A^{n}$ the set of words of length $n$ that appear in $X$. The subshift $(X, \sigma)$ is transitive if and only if for every $u, v \in \mathcal{L}(X)$ there exists $w \in \mathcal{L}(X)$ such that $u w v \in \mathcal{L}(X)$. The entropy of $(X, \sigma)$ is the exponential growth rate of the number of appearing words as the length of the words increases:

$$
h(X, \sigma)=\lim _{n \rightarrow \infty} \frac{1}{n} \log _{2}\left(\left|\mathcal{L}_{n}(X)\right|\right)
$$

We only need the following facts about entropy.

Proposition 1 ([13, Proposition 4.1.9.]). Let $X$ and $Y$ be subshifts and $F: X \rightarrow Y$ a factor map. Then $h(Y, \sigma) \leq h(X, \sigma)$.

Proposition 2 ([13, Corollary 4.4.9.]). Let $X$ be a transitive sofic shift and $Y \subseteq X$ a subshift. If $Y \neq X$, then $h(Y, \sigma)<h(X, \sigma)$. 
Cellular automata A cellular automaton $(C A)$ is a dynamical system $\left(A^{\mathbb{M}}, F\right)$ where $F$ is a shift-commuting map, i.e. $F \sigma=\sigma F$. When $\mathbb{M}=\mathbb{N}$, the CA is called one-sided and when $\mathbb{M}=\mathbb{Z}$, the $\mathrm{CA}$ is called two-sided. We will often refer to a CA by the function name alone, i.e. talk about the CA $F$. The CA $F$ is reversible if there exists another $\mathrm{CA} F^{\prime}$ such that $F^{\prime} F(c)=c=F F^{\prime}(c)$ for all $c \in A^{\mathbb{M}}$. Let $D=[i, j] \subset \mathbb{M}$ and let $G_{l o c}: A^{D} \rightarrow A$. Define $G: A^{\mathbb{M}} \rightarrow A^{\mathbb{M}}$ by $G(c)_{i}=G_{l o c}\left(\left(\sigma^{i}(c)\right)_{D}\right)$. Then $G$ is continuous and commutes with $\sigma$, so it is a cellular automaton. The set $D$ is a local neighborhood of $G$ and the function $G_{l o c}$ is a local rule of $G$. According to the Curtis-Hedlund-Lyndon Theorem every CA is defined by a local rule. Let $m, a \in \mathbb{Z}$ be the smallest numbers such that $G$ is defined by a local rule on $A^{[-m, a]}$, then $m$ is the memory, $a$ the anticipation, and $r=\max \{a, m\}$ the radius of $G$. If $m \leq 0$ then $G$ is memoryless. We overload the notation for the global function and use it also on finite words: For any $n>2 r$ and $u \in A^{n}$ we define in a natural way the word $G(u) \in A^{n-2 r}$.

For a $\mathrm{CA}\left(A^{\mathbb{Z}}, F\right)$ the space-time diagrams are the two-way infinite orbits denoted as

$$
s t(F)=\left\{\left(c^{(i)}\right)_{i \in \mathbb{Z}} \in\left(A^{\mathbb{Z}}\right)^{\mathbb{Z}} \mid \forall i: F\left(c^{(i)}\right)=c^{(i+1)}\right\} .
$$

As a pictorial presentation we consider these as coloured square lattices where rows are the points of the orbit and time advances downwards; left, right, up, and down should be understood accordingly. Notice that our space-time diagrams present bi-infinite orbits, so only the configurations in the limit set $\Lambda_{F}=\bigcap_{n \in \mathbb{N}} F^{n}\left(A^{\mathbb{Z}}\right)$ of $F$ are considered. However our results will be for surjective cellular automata, in which case $\Lambda_{F}=A^{\mathbb{Z}}$.

Let $\left(A^{\mathbb{M}}, F\right)$ be a $\mathrm{CA}$. Then $F$ is positively expansive if there exists $\varepsilon>0$ such that

$$
\forall c, e \in A^{\mathbb{M}}:\left(c \neq e \Longrightarrow\left(\exists n \in \mathbb{N}: d\left(F^{n}(c), F^{n}(e)\right)>\varepsilon\right)\right) .
$$

If $F$ is reversible, then $F$ is expansive if there exists $\varepsilon>0$ such that

$$
\forall c, e, \in A^{\mathbb{M}}:\left(c \neq e \Longrightarrow\left(\exists n \in \mathbb{Z}: d\left(F^{n}(c), F^{n}(e)\right)>\varepsilon\right)\right) .
$$

If $F$ is reversible and $\mathbb{M}=\mathbb{Z}$, then $F$ is right-expansive if there exists $\varepsilon>0$ such that

$$
\forall c, e \in A^{\mathbb{Z}}:\left(\left(\exists i>0: c_{i} \neq e_{i}\right) \Longrightarrow\left(\exists n \in \mathbb{Z}: d\left(F^{n}(c), F^{n}(e)\right)>\varepsilon\right)\right) .
$$

Left-expansive and positively right/left-expansive are defined analogously.

Remark 1. A one-sided cellular automaton $\left(A^{\mathbb{N}}, F\right)$ can be naturally extended to a memoryless two-sided cellular automaton $\left(A^{\mathbb{Z}}, F\right)$. One should be slightly careful with this: Firstly notice that $F$ may be reversible over $A^{\mathbb{Z}}$ but nonreversible over $A^{\mathbb{N}}$, for example the shift map. (However if $F$ is reversible over $A^{\mathbb{N}}$, then its extension to $A^{\mathbb{Z}}$ is also reversible.) Secondly, if $\left(A^{\mathbb{N}}, F\right)$ is expansive, then $\left(A^{\mathbb{Z}}, F\right)$ is right-expansive (but never expansive). Similarly for positively expansive cellular automata. 


\section{$3 \quad$ Ultimate expansivity and trace subshifts}

We generalize the concept of expansivity to possibly non-reversible cellular automata. A cellular automaton $\left(A^{\mathbb{Z}}, F\right)$ is ultimately expansive if there exists $\varepsilon>0$ such that

$$
\forall\left(c^{(i)}\right)_{i \in \mathbb{Z}},\left(e^{(i)}\right)_{i \in \mathbb{Z}} \in \operatorname{st}(F):\left(c^{(0)} \neq e^{(0)} \Longrightarrow\left(\exists n \in \mathbb{Z}: d\left(c^{(n)}, e^{(n)}\right) \geq \varepsilon\right)\right)
$$

Ultimately right-expansive and ultimately left-expansive are defined the same way. The set of ultimately expansive cellular automata contains the expansive cellular automata, the positively expansive cellular automata, and more.

Example 1. A cellular automaton $\left(A^{\mathbb{Z}}, F\right)$ is nilpotent if there exists $q \in A$ and $n \in \mathbb{N}$ such that for every $c \in A^{\mathbb{Z}}$ we have that $F^{n}(c)=\cdots q q q \cdots$. Then $\tau(F)$ is a singleton and it follows that $F$ is ultimately expansive.

There cannot be a reversible cellular automaton that would be ultimately expansive but not expansive, as ultimate expansivity and reversibility together imply expansivity. However there are surjective ultimately expansive cellular automata which are neither expansive nor positively expansive:

Example 2. Let $A=\{0,1\}, \sigma: A^{\mathbb{Z}} \rightarrow A^{\mathbb{Z}}$ be the shift map and $\mathcal{X}: A^{\mathbb{Z}} \rightarrow A^{\mathbb{Z}}$ be the xor-CA, that is the CA defined by $\mathcal{X}_{l o c}: A^{[-1,1]} \rightarrow A, \mathcal{X}_{l o c}(a b c)=a \oplus c$, where $\oplus$ denotes addition modulo 2. The shift map $\sigma$ is expansive, and so also ultimately expansive. The xor-CA cannot be expansive as it is not reversible, however it is positively expansive, and so also ultimately expansive. Consider the direct product of these, that is the CA $\sigma \times \mathcal{X}: A^{\mathbb{Z}} \times A^{\mathbb{Z}} \rightarrow A^{\mathbb{Z}} \times A^{\mathbb{Z}}$ defined by $\sigma \times \mathcal{X}(c, e)=(\sigma(c), \mathcal{X}(e))$. This is neither expansive (not even reversible) nor positively expansive (since $\sigma$ is not). However $\sigma \times \mathcal{X}$ is ultimately expansive.

In what follows we will consider configurations of $\left(A^{n}\right)^{\mathbb{Z}}$ where $n>0$, which can lead to indexing issues. To avoid these issues we define for every $n \in \mathbb{N} \backslash$ $\{0\}, i \in\{0, \ldots, n-1\}$, and $j \in\{0, \ldots, i\}$ projection functions $\pi_{i}: A^{n} \rightarrow A$ and $\pi_{[i, j]}: A^{n} \rightarrow A^{j-i+1}$ where $\pi_{i}\left(a_{0} \cdots a_{n-1}\right)=a_{i}$ and $\pi_{[i, j]}\left(a_{0} \cdots a_{n-1}\right)=a_{i} \cdots a_{j}$. We also extend these to $\left(A^{n}\right)^{\mathbb{Z}}$ cell-wise.

Let $\left(A^{\mathbb{Z}}, F\right)$ be a cellular automaton with radius $r$. The $n$-trace of $F$ is the two-sided subshift of columns of width $n$ that appear in the space-time diagrams of $F$, i.e.

$$
\tau_{n}(F)=\left\{t \in\left(A^{n}\right)^{\mathbb{Z}} \mid \exists\left(c^{(i)}\right)_{i \in \mathbb{Z}} \in \operatorname{st}(F): \forall j \in \mathbb{Z}: t_{j}=c_{[0, n)}^{(j)}\right\}
$$

Since we are mainly interested in the $2 r$-trace, we denote $\tau(F)=\tau_{2 r}(F)$ and call it the trace of $F$. Notice that our traces are always two-sided subshifts, which is in line with our definition of space-time diagrams: Traces are vertical stripes of space-time diagrams.

A cellular automaton $\left(A^{\mathbb{Z}}, F\right)$ is ultimately right-expansive if and only if there exists $n \in \mathbb{N}$ such that for all $\left(c^{(i)}\right)_{i \in \mathbb{Z}},\left(e^{(i)}\right)_{i \in \mathbb{Z}} \in s t(F)$ it holds that

$$
\left(\forall j \in\{0, \ldots, 2 n\}: c_{[0,2 r)}^{(j)}=e_{[0,2 r)}^{(j)}\right) \Longrightarrow c_{2 r}^{(n)}=e_{2 r}^{(n)} .
$$




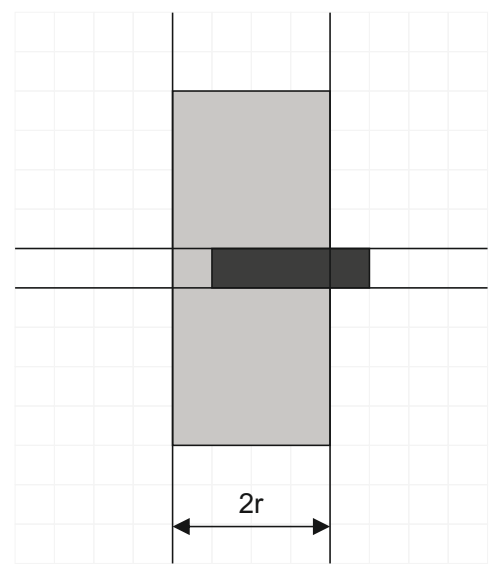

Fig. 1. Ultimately right-expansive CA defines a CA $\vec{F}$ which draws the (right halves) of the space-time diagrams. The figure illustrates how $\vec{F}$ is defined; assuming that the grid has a valid space-time diagram of $F$, then $\vec{F}$ maps the pattern in the light gray area to the pattern in the dark gray area.

So for an ultimately right-expansive $F$ we can define a CA $\left(\left(A^{2 r}\right)^{\mathbb{Z}}, \vec{F}\right)$ such that for every $t \in \tau(F)$ we have that $\vec{F}(t) \in \tau(F)$ is the unique configuration such that $\pi_{[1,2 r)}(t)=\pi_{[0,2 r-1)}(\vec{F}(t))$ and the last column of $\vec{F}(t)$ is the column defined by (4) (Figure 1). How $\vec{F}$ maps the words that do not appear in $\tau(F)$ is irrelevant for us. Then $\left\{\left(\pi_{0}\left(\vec{F}^{i}(t)\right)\right)_{i \in \mathbb{N}} \mid t \in \tau(F)\right\}$ is the set of right halves of $\operatorname{st}(F)$.

Next we consider $\varepsilon$-chains for cellular automata. Let $\left(A^{\mathbb{Z}}, F\right)$ be a CA. For every $n \in \mathbb{N} \backslash\{0\}$ we define a directed labeled multigraph $\mathcal{G}_{n}(F)=\left(V_{n}, E_{n}\right)$ as follows:

- The set of vertices is $V_{n}=A^{n}$.

- For every $u \in V_{n}$ and $x, y \in A^{r}$ there is a labeled edge $u \stackrel{x y}{\longrightarrow} F(x u y)$ in $E_{n}$.

The graph $\mathcal{G}_{n}(F)$ defines an $\operatorname{SFT} X_{n}(F) \subseteq\left(A^{n}\right)^{\mathbb{Z}}$ where $(u, v) \in\left(A^{n}\right)^{2}$ is forbidden if there is no edge $u \longrightarrow v$. The points of $X_{n}(F)$ are essentially the pseudo-orbits of $F$. From the definitions we get a characterization of chain transitive CA's that is more convenient for us:

Proposition 3. A $C A\left(A^{\mathbb{Z}}, F\right)$ is chain-transitive if and only if $X_{n}(F)$ is transitive for every $n$. 
On Dynamical Complexity of Surjective Ultimately Right-Expansive CA

Proof.

$\left(A^{\mathbb{Z}}, F\right)$ is chain-transitive

$$
\begin{aligned}
& \Longleftrightarrow \forall \varepsilon>0: \forall c, e \in A^{\mathbb{Z}}: \exists c=c^{(0)}, \ldots, c^{(n)}=e: d\left(F\left(c^{(i)}\right), c^{(i+1)}\right)<\varepsilon \\
& \Longleftrightarrow \forall k \in \mathbb{N}: \forall c, e \in A^{\mathbb{Z}}: \exists c=c^{(0)}, \ldots, c^{(n)}=e: F\left(c^{(i)}\right)_{[-k, k]}=\left(c^{(i+1)}\right)_{[-k, k]} \\
& \Longleftrightarrow \forall k \in \mathbb{N}: \mathcal{G}_{k}(F) \text { is strongly connected } \\
& \Longleftrightarrow \forall k \in \mathbb{N}: X_{k}(F) \text { is transitive. }
\end{aligned}
$$

For every $n \geq 2 r$ and $i \in\{0, \ldots, n-2 r\}$ we denote $\Sigma_{i, n}(F)=\pi_{[i, i+2 r)}\left(X_{n}(F)\right)$. Subshifts $\Sigma_{i, n}(F)$ are sofic as factors of SFTs. The elements of $\Sigma_{i, n}(F)$ can be considered as such configurations that can be extended with $i$ columns to the left and with $n-i-2 r$ columns to the right without introducing violations of the local rule of $F$. Our chief interest are the middle columns, that is the subshifts $\Sigma_{n-r, 2 n}(F)$, so we denote them by $\Sigma_{n}(F)$ and call them approximations of the trace. We simplify the notations and omit the name of the CA when it is clear from the context.

We notice that the approximations $\Sigma_{n}$ coincide with $\tau(F)$ in the limit:

Proposition 4. Let $\left(A^{\mathbb{Z}}, F\right)$ be a surjective $C A$ with radius $r$. Then $\tau(F)=$ $\bigcap_{n \geq r} \Sigma_{n}$.

Proof. " $\subseteq$ ": If $t \in \tau(F)$ then any space-time diagram that contains $t$ shows that $t \in \Sigma_{n}$ for every $n \in \mathbb{N}$ where $n \geq r$.

"D": Let $t \in \bigcap_{n \geq r} \Sigma_{n}$, and suppose that $t \notin \tau(F)$. Then $t$ must contain some forbidden word of the subshift $\tau(F)$, i.e. there exists $i, j \in \mathbb{Z}$ such that $t_{[i, j]} \notin \mathcal{L}(\tau(F))$. But this is a contradiction, since $\mathcal{L}_{k}\left(\Sigma_{k r}\right)=\mathcal{L}_{k}(\tau(F))$ for all $k \in \mathbb{N}$.

The pseudo-orbit tracing property has the following characterization for cellular automata.

Proposition 5. Let $\left(A^{\mathbb{Z}}, F\right)$ be a surjective $C A$. Then $F$ has the pseudo-orbit tracing property if and only if there exists $n$ such that $\tau(F)=\Sigma_{n}$.

Proof. " $\Rightarrow$ ": The pseudo-orbit tracing property immediately implies that there exists $n \in \mathbb{N}$ such that the set of middle columns of $X_{2 n}$ is $\tau(F)$.

" $\Leftarrow$ ": If $\tau(F)=\Sigma_{n}$ then for any $k$ and any $x \in X_{2 k+2 n}$ there exists an orbit $\left(c^{(i)}\right)_{i \in \mathbb{Z}}$ such that $c_{[-k, k]}^{(i)}$ is the word of length $2 k$ in the middle of $x_{i}$ for every $i \in \mathbb{Z}$.

From this it follows that if $F$ has the pseudo-orbit tracing property, then $\tau_{n}(F)$ is sofic for every $n$. This was already proved by Kưrka in [10] where it was also proved that if every $\tau_{n}(F)$ is an SFT, then $F$ has the pseudo-orbit tracing property. 
We say that $F$ has the left-extension property if there exists $i$ and $N$ such that

$$
\forall n \geq N, m \geq 0: \Sigma_{i, n}=\Sigma_{i+m, n+m} .
$$

The right-extension property is defined analogously. From Proposition 5 we see that the extension properties are one-sided variants of the pseudo-orbit tracing property, i.e. that a cellular automaton has the pseudo-orbit tracing property if and only if it has both the right- and the left-extension property.

Remark 2. A memoryless CA $F$ with neighborhood [0, $r]$ has the left-extension property: Take any configuration $t \in\left(A^{r}\right)^{\mathbb{Z}}$ and any $a \in A$. Define configurations $\left(t^{(i)}\right)_{i \in \mathbb{N}}$ as follows:

$$
t_{j}^{(i)}= \begin{cases}a \pi_{[1, r)}\left(t_{j}\right), & \text { if } j \leq-i \\ F_{l o c}\left(\pi_{0}\left(t_{j-1}^{(i)}\right) t_{j-1}\right) \pi_{[1, r)}\left(t_{j}\right) & \text { if } j>-i\end{cases}
$$

By compactness the sequence $\left(t^{(i)}\right)_{i \in \mathbb{N}}$ has a converging subsequence $\left(t^{(i)}\right)_{i \in \mathcal{I}}$. Let the limit of this sequence be $t^{\prime}$. Now $t^{\prime}$ is a configuration that shows that $t$ can be extended with one column to the left. We can repeat the process to extend $t$ to the left as much as we will. This shows that $\Sigma_{0, n}=\Sigma_{m, n+m}$ for all $n \geq r, m \geq 0$.

Now the following corollary is immediate.

Corollary 1. If $F$ is memoryless, then $\tau(F)=\bigcap_{i \geq 2 r} \Sigma_{0, i}$.

Proof. From Remark 2 it follows that for all $n \geq r$ we have $\Sigma_{0, n}=\Sigma_{n-r}$, and so the claim follows from Proposition 4.

\section{Surjective ultimately right-expansive CA are chain-transitive}

Consider a CA $\left(A^{\mathbb{Z}}, F\right)$. Let $P=\left\{A_{i}\right\}_{i \in\{0, \ldots, n-1\}}$ be a partition of $A$. We say that $F$ respects $P$ at $x \in A^{\mathbb{Z}}$ if for every $k \in \mathbb{N}$ there exists $j \in\{0,1, \ldots, n-1\}$ such that $F^{k}(x) \in A_{j}^{\mathbb{Z}}$. Let $S$ be the set of all points where $F$ respects $P$. This is a subshift. Now we define a projection $\iota:\left(A^{\mathbb{Z}}\right)^{\mathbb{N}} \rightarrow\left((\{0,1, \ldots, n-1\})^{\mathbb{Z}}\right)^{\mathbb{N}}$ by $\iota(c)_{i, j}=k$ if $c_{i, j} \in A_{k}$. Next we project the forward orbits of $S$ cell-wise using $\iota$. According to the definition of $S$, each row is constant after this mapping, so we can consider the projected forward orbits as a one-sided one-dimensional subshift over the alphabet $\{0,1,2, \ldots, n-1\}$. We will call this subshift the stripe trace of $F$ with respect to $P$.

Lemma 1. Let $\left(A^{\mathbb{Z}}, F\right)$ be a $C A$ and $P=\left\{A_{i}\right\}_{i \in\{0, \ldots, n-1\}}$ a non-trivial partition of $A$. The stripe trace of $F$ with respect to $P$ cannot be the full shift $\{0,1, \ldots, n-$ $1\}^{\mathbb{N}}$. 
Proof. We can simplify the situation and assume that the local rule of $F$ is a function $A^{[0,1]} \rightarrow A$ by composing $F$ with a suitable power of the shift map and using a suitable grouping map $g_{n}: A^{\mathbb{Z}} \rightarrow\left(A^{n}\right)^{\mathbb{Z}},\left(g_{n}(c)\right)_{i}=c_{i n} c_{i n+1} \cdots c_{i n+n-1}$. This can be done since shifting does not change the stripe trace and we can define a partition of the grouped alphabet that gives the same stripe trace. We may also assume that our partition is $\left\{A_{0}, A_{1}\right\}$.

Suppose that the stripe trace of $F$ is the full shift $\{0,1\}^{\mathbb{N}}$. Let $S$ be the set of configurations that respect the partition $\left\{A_{0}, A_{1}\right\}$. For every $l \in \mathbb{N} \backslash\{0\}$ and $u \in\{0,1\}^{l}$ we denote

$$
S_{u}=\left\{v \in \mathcal{L}_{l}(S) \mid F^{j}(v)_{0} \in A_{u_{j}} \text { for all } j \in\{0, \ldots, l-1\}\right\} .
$$

Since the stripe trace is the full shift, all of these $S_{u}$ sets must be non-empty. Let $|A|=k$. We will show that no matter how large $k$ is, it will not be large enough.

Let $u, v \in A^{l}$ for some $l \in \mathbb{N} \backslash\{0\}$. Now consider a word $w \in S_{u v}$. Let $w=w^{\prime} w^{\prime \prime}$ where $w^{\prime}, w^{\prime \prime} \in A^{l}$. From the definition of $S_{u v}$ we have that both $w^{\prime}$ and $w^{\prime \prime}$ must be in $S_{u}$, so we have $S_{u v} \subseteq S_{u} S_{u}$ and so

$$
\bigcup_{v \in A^{l}} S_{u v} \subseteq S_{u} S_{u} .
$$

From this we get the inequality $\sum_{v \in A^{l}}\left|S_{u v}\right| \leq\left|S_{u}\right|^{2}$, and then we have that

$$
\min _{v \in A^{l}}\left|S_{u v}\right| \leq \frac{\left|S_{u}\right|^{2}}{2^{l}} .
$$

From this we get an upper bound for the size of the smallest set $S_{u_{1} \cdots u_{2}{ }^{n}}$ for every $n \in \mathbb{N}$ :

$$
\min _{u_{1} u_{2} \cdots u_{2^{n}} \in A^{2^{n}}}\left|S_{u_{1} u_{2} \cdots u_{2} n}\right| \leq z_{n},
$$

where $z_{n}$ is defined by the recursive formula

$$
z_{n+1}=\frac{z_{n}^{2}}{2^{2^{n}}}, z_{0}=\frac{k}{2} .
$$

We get this from the fact that $\min \left\{\left|S_{0}\right|,\left|S_{1}\right|\right\} \leq \frac{k}{2}$, and then using inequality (5). Solving this recursion yields

$$
z_{n}=\frac{k^{2^{n}}}{2^{2^{n}+n \cdot 2^{n-1}}} .
$$

But now we see that the sequence $\left(z_{n}\right)_{n \in \mathbb{N}}$ converges to zero, and so for some $N \in \mathbb{N}$ and $u \in\{0,1\}^{N}$ we have that $S_{u}=\emptyset$ which proves the claim.

Since subshifts with only periodic points are finite, one can easily obtain these as stripe traces by using only uniform configurations. Using the infinite firing squad CA [8] we see that also the sunny side up subshift, i.e. $\left\{c \in\{0,1\}^{\mathbb{N}}\right.$ | 
$c$ has at most one 1$\}$, is a stripe trace of a $\mathrm{CA}$. There are some more or less trivial generalizations of these examples. We also have an example of a stripe trace configuration that is not eventually periodic. However we believe, for basically the same reason as why the full shift cannot be a stripe trace, that stripe traces always have zero entropy.

While the stripe trace has certain interest in itself, we just need the above lemma as a technical detail in the following.

Theorem 1. A surjective ultimately right-expansive $C A\left(A^{\mathbb{Z}}, F\right)$ is chain-transitive.

Proof. According to Proposition 3, we can equally well show that the subshifts $X_{n}(F)$ are transitive, i.e. that their defining graphs $\mathcal{G}_{n}(F)$ are strongly connected. Clearly if $X_{n}(F)$ is transitive, then so is $X_{k}(F)$ for any $k<n$ so we can assume that $n \geq 2 r$.

Let us remind that the vertex set of $\mathcal{G}_{n}(F)$ is $V=A^{n}$ and that we have an edge $u \stackrel{x y}{\longrightarrow} v$ if and only if $F(x u y)=v$, where $x, y \in A^{r}$. Let $\operatorname{deg}_{i n}(u)$ denote the number of incoming edges to the vertex $u$ and $\operatorname{deg}_{\text {out }}(u)$ the number of outgoing edges from the vertex $u$. By definition we have that $\operatorname{deg}_{\text {out }}(u)=|A|^{2 r}$ for every $u \in V$. By the balancedness property of surjective CA's [15] we also have that $\operatorname{deg}_{\text {in }}(u)=|A|^{2 r}$ for every $u \in V$. It is well-known that $\operatorname{deg}_{\text {in }}(u)=\operatorname{deg}_{\text {out }}(u)$ for every $u \in V$ if and only if the graph is a union of Eulerian graphs, that is graphs which have a directed path (sequence of edges traversed in the direction of the edges) where every edge appears exactly once, and which starts and ends at the same vertex. Especially we have that there are no edges between the strongly connected components of $\mathcal{G}_{n}(F)$.

Let $\mathcal{G}_{n}(F)_{1}, \mathcal{G}_{n}(F)_{2}, \ldots, \mathcal{G}_{n}(F)_{m}$ be the strongly connected components of $\mathcal{G}_{n}(F)$ and $V_{1}, \ldots, V_{m} \subseteq A^{n}$ the corresponding vertex sets. Now $\left\{V_{i}\right\}_{i \in\{1, \ldots, m\}}$ is a partition of $A^{n}$. There are no edges between the strongly connected components which means that the pseudo-orbits cannot go from a cylinder of the set $V_{i}$ to a cylinder of the set $V_{j}$ for any $i \neq j$. Then definitely the orbits cannot do this either, and so we have that $\tau_{n}(F) \subseteq \bigcup_{i \in\{1, \ldots, m\}} V_{i}^{\mathbb{Z}}$. Notice that we have not yet used the ultimate right-expansivity, so everything said thus far holds for surjective cellular automata in general. Since $F$ is ultimately rightexpansive we have a cellular automaton $\left(\left(A^{n}\right)^{\mathbb{Z}}, \vec{F}_{n}\right)$ which is just a natural extension of $\left(\left(A^{2 r}\right)^{\mathbb{Z}}, \vec{F}\right)$. Now we consider the cellular automaton $\vec{F}_{n}^{n}$, so that the preimages and images are not overlapping stripes of space-time diagrams. Each of these stripes is contained in $V_{i}^{\mathbb{Z}}$ for some $i \in\{1, \ldots, m\}$. If $m>1$ then $\vec{F}_{n}^{n}$ and the partition $\left\{V_{i}\right\}_{\{1, \ldots, m\}}$ would contradict Lemma 1: Take an arbitrary $c \in\left(A^{n}\right)^{\mathbb{Z}}=\left(\bigcup_{i \in\{1, \ldots, m\}} V_{i}\right)^{\mathbb{Z}}$. Let $s_{c} \in \operatorname{st}(F)$ be such that $c$ is a row in $s_{c}$. Now applying $\vec{F}_{n}^{n}$ to a stripe of width $n$ in $s_{c}$ shows that we can draw an arbitrary stripe trace configuration over $\{1, \ldots, m\}$ with $\vec{F}_{n}^{n}$. Notice that the way we complete $\vec{F}$ to the full shift is irrelevant since this can only grow the stripe trace. This concludes the proof.

This has the following immediate corollary. 
Corollary 2. Let $\left(A^{\mathbb{Z}}, F\right)$ be a surjective ultimately right-expansive $C A$. Then $\Sigma_{i, n}(F)$ is transitive for every $i, n \in \mathbb{N}$ such that $i \leq n-2 r$.

Proof. According to Proposition 1 we have that $X_{n}(F)$ is transitive for every $n \in \mathbb{N}$. The claim follows immediately since a factor of a transitive system is also transitive.

\section{Traces of expansive cellular automata}

Next we provide an elementary proof that surjective ultimately right-expansive cellular automata with the left-extension property have the pseudo-orbit tracing property. This is almost the same result as Nasu's [18, Theorem 6.3]. The main differences are that our proof is only for cellular automata over full shifts while Nasu's is over transitive SFT's, and on the other hand that Nasu has an additional assumption of chain-recurrence, which Nasu uses to guarantee chaintransitivity.

Theorem 2. Let $\left(A^{\mathbb{Z}}, F\right)$ be a surjective ultimately right-expansive $C A$ with the left extension property. Then $F$ has the pseudo-orbit tracing property and $\tau(F)$ is a transitive sofic shift. If $F$ is memoryless, then $\tau(F)$ is an SFT.

Proof. We have seen that $\Sigma_{l}(F)$ is a sofic shift for every $l \in \mathbb{N}$, and according to Corollary 2 it is also transitive. We will show that there exists $m \in \mathbb{N}$ such that $\tau(F)=\Sigma_{m}$.

Let $i, N \in \mathbb{N}$ be given by the left-extension property, so that for all $n \geq N$ and $m \geq 0$ we have that $\Sigma_{i, n}=\Sigma_{i+m, n+m}$. Let $\vec{F}:\left(A^{2 r}\right)^{\mathbb{Z}} \rightarrow\left(A^{2 r}\right)^{\mathbb{Z}}$ be a cellular automaton defined by the ultimate right-expansivity of $F$ (how $\vec{F}$ maps words that do not appear in $\tau(F)$ is irrelevant). As $\mathcal{L}\left(\Sigma_{k r}\right)=\mathcal{L}_{k}(\tau(F))$ we have that there exists $m^{\prime}$ such that $\vec{F}$ is defined on $\Sigma_{m^{\prime}}$. Let $m \geq \max \left\{m^{\prime}, N-i\right\}$.

Claim: $\vec{F}\left(\Sigma_{m}\right)=\Sigma_{m-1}$.

Proof: "ᄃ": Let $t^{(0)} \in \Sigma_{m}$. Since $t^{(0)}$ is the central stripe of some element in $X_{m}(F)$ there is a $t^{(1)} \in \Sigma_{m-r+1,2 m} \subseteq \Sigma_{m-1}$ such that $t^{(0,1)} \in\left(A^{2 r+1}\right)^{\mathbb{Z}}$ defined by

$$
\pi_{[0,2 r)}\left(t^{(0,1)}\right)=t^{(0)} \text { and } \pi_{[1,2 r]}\left(t^{(0,1)}\right)=t^{(1)}
$$

has no violations of the local rule of $F$. But then it has to be that $\vec{F}\left(t^{(0)}\right)=$ $t^{(1)}$.

" $\supseteq$ ": Now let $t^{(1)} \in \Sigma_{m-1}$. By the left-extension property we have that

$t^{(1)} \in \Sigma_{m-r+1,2 m}$, so that there exists $t^{(0)} \in \Sigma_{m}$ such that $\vec{F}\left(t^{(0)}\right)=t^{(1)}$.

Now we have that $\Sigma_{m-1}$ is a factor of $\Sigma_{m}$, so $\Sigma_{m-1}$ has at most the entropy of $\Sigma_{m}$ (Proposition 1). But we also have that $\Sigma_{m} \subseteq \Sigma_{m-1}$, and so $\Sigma_{m}$ and $\Sigma_{m-1}$ have the same entropy. Since $\Sigma_{m-1}$ is a transitive subshift, all its proper subshifts have strictly smaller entropy (Proposition 2), and so it follows that $\Sigma_{m}=\Sigma_{m-1}$. Let $l=\max \left\{m^{\prime}, N-i\right\}$ and we have $\tau(F)=\Sigma_{l}$. Now $F$ has the pseudo-orbit tracing property according to Proposition 5. 
It is left to show that if $F$ is memoryless, say with neighborhood $[0, r]$, then $\tau(F)$ is an SFT. According to Corollary 1 we now have that $\Sigma_{0, k+r}=\tau(F)$ for some $k$. Let the radius of $\vec{F}$ be $z$. Apply $\vec{F}$ to the words $u \in\left(A^{r}\right)^{k \cdot 2 z+1}$ for $k$ times. If no violations of the local rule of $F$ appears, then $u \in \mathcal{L}\left(\Sigma_{m}\right)=\mathcal{L}(\tau(F))$. On the other hand, if a violation does appear, then $u \notin \mathcal{L}(\tau(F))$. We get a finite set of forbidden words that defines $\tau(F)$.

Remark 3. Theorem 2 implies the following:

- If $\left(A^{\mathbb{N}}, F\right)$ is surjective and positively expansive, then $\tau(F)$ is an SFT ([10], improved in [4])

- If $\left(A^{\mathbb{N}}, F\right)$ is reversible and expansive, then $\tau(F)$ is an SFT ([17])

- If $\left(A^{\mathbb{Z}}, F\right)$ is reversible, (right-)expansive, and memoryless, then $\tau(F)$ is an $\operatorname{SFT}([18])$.

\section{Right-expansive cellular automaton with non-sofic trace}

Next we show that while it might be possible to replace the assumption of left-extension property in Theorem 2 with a weaker assumption, it can not be dropped entirely. Using the construction from [9] we give an example of a rightexpansive CA that has a non-sofic trace. The next paragraphs summarizes what we need, but for a more detailed presentation we refer the reader to [7], [9], and [14]. Notice that our example is over a full shift, which in particular is a transitive subshift; an example by Fiebig of an expansive cellular automaton over non-transitive subshift was already presented in the appendix of [1].

A set of Wang tiles $T$ is a set of squares with each edge coloured with a colour from a finite colour set $A$. Let us fix that $(x, y, z, w) \in A^{4}$ is a Wang tile such that the colours are presented in the order W-N-E-S (west-north-east-south). A tiling by $T$ is an assignment $\mathbb{Z}^{2} \rightarrow T$. A tiling is valid if the adjacent edges have the same colour. The set $T$ is called $N W$-deterministic if for all $a, b \in A$ we have that $|\{a\} \times\{b\} \times A \times A \cap T| \leq 1$. Other XY-determinism's are defined analogously. If $T$ is both NW- and SE-deterministic, then $T$ is called two-way deterministic. Any two-way deterministic tile set can be completed in the sense that we can add tiles $T^{C}$ so that for all $a, b \in A$ holds that $\left|\{a\} \times\{b\} \times A \times A \cap\left(T \cup T^{C}\right)\right|=$ $1=\left|A \times A \times\{a\} \times\{b\} \cap\left(T \cup T^{C}\right)\right|$. This can be done since we must be missing the same number of NW- and SE-pairs; in $T^{C}$ we just match these arbitrarily. This means that a two-way deterministic set of Wang tiles $T$ can be used to define a reversible cellular automaton $F_{T}:\left(T \cup T^{C}\right)^{\mathbb{Z}} \rightarrow\left(T \cup T^{C}\right)^{\mathbb{Z}}$ where configurations represent SW-NE-diagonals of valid tilings with $T \cup T^{C}$.

A tiling $c$ by a Wang tile set $T$ is periodic if there exits $(x, y) \in \mathbb{Z}^{2} \backslash\{(0,0)\}$ such that for every $(i, j) \in \mathbb{Z}^{2}$ we have that $c_{i, j}=c_{i+x, j+y}$. A Wang tile set $T$ is aperiodic if it admits a valid tiling, but none of the valid tilings is periodic. We need the fact that there exists an aperiodic two-way deterministic Wang tile set. Such do exist: The Wang tile set derived from Amman's aperiodic tile set [5] is 
such, and the tile set constructed in [14] is even 4-way deterministic. Details of the chosen tile set are irrelevant for us.

Proposition 6. There exists a right-expansive cellular automaton with a nonsofic trace.

Proof. Following paragraph shortly describes what we get from a construction in $[9]$ :

Let $T$ be a two-way deterministic aperiodic set of Wang tiles. Define $F_{T}$ : $\left(T \cup T^{C}\right) \rightarrow\left(T \cup T^{C}\right)$ as above; the tiles in $T^{C}$ are considered to be tiling errors. For large enough $k$ it holds that $\sigma^{k} F_{T}$ is expansive. Denote $F=\sigma^{k} F_{T}$. Next signals are added: Let $G:\left(\left(T \cup T^{C}\right) \times\{0,1\}^{2}\right)^{\mathbb{Z}} \rightarrow\left(\left(T \cup T^{C}\right) \times\{0,1\}^{2}\right)^{\mathbb{Z}}$ where the tiling-layer is mapped by $F$ and the signal layer is mapped as illustrated by Figure 2. As noted in [9] this $G$ is right-expansive and further has the property that if there is a tiling error somewhere, then every column right of the tiling error will contain both zero- and one-signals.
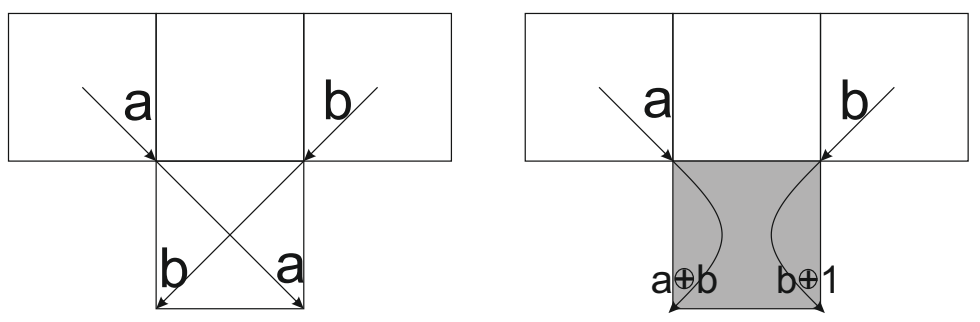

Fig. 2. Here $a, b \in\{0,1\}$, the light gray represents a tiling error, and $\oplus$ denotes addition modulo 2. The label of the arrow pointing to the lower left corner is the first bit and the label of the arrow pointing to the lower right corner is the second bit.

Let $r$ be the radius of $G$ and suppose that $\tau(G)$ is sofic. Take a spacetime diagram which on the tiling-layer contains only states from $T$ and on the signal-layer all the signals are zeroes. Such exists as $T$ admits valid tilings and then setting every signal to zero will give a suitable space-time diagram. Let $t \in\left((T \times\{(0,0)\})^{2 r}\right)^{\mathbb{Z}}$ be a column of this space-time diagram. By soficness it follows that there exists $i \in \mathbb{Z}, n \in \mathbb{N}$ such that $u=t_{[i, i+n)}$ is such that $\cdots$ uиu $\cdots \in \tau(G)$. Now since the tiling-layer is expansive, this implies that in the space-time diagram that has $\cdots u u u \cdot$ as a column, the tiling layer must be periodic. But since $T$ is an aperiodic tile set, it then has to be that there are tiling errors densely; that is to say that there exists $m \in \mathbb{N}$ such that every $m \times m$ square in the space-time diagram has a cell whose tiling-layer is in a state from $T^{C}$. In particular there has to be tiling errors left of the column $\cdots u u u \cdots$. But this is a contradiction, since if there is a tiling error left of the column, then the column's signal-layer has both zeroes and ones, but $\cdots$ uuu $\cdots$ has only zeroes. 


\section{Conclusion}

To conclude let us give some overview of what is known and what is still open. Two main concepts we considered were ultimate expansivity and the pseudoorbit tracing property. For one-dimensional cellular automata these concepts have one-sided variants, namely left- and right-expansivity, and left- and rightextension property. In this setting we have asked what can be said about the trace of a cellular automaton assuming different combinations of expansivity and extension properties. For surjective cellular automata the following are known:

- If $F$ has both the left- and the right-extension property, then the trace of $F$ is sofic (from definitions).

- If $F$ has the left-extension property and is right-expansive, then the trace of $F$ is sofic (Theorem 2).

- If $F$ is right-expansive, then the trace of $F$ is not necessarily sofic (Proposition 6).

- If $F$ has the left-extension property, then $F$ is not necessarily sofic (in [6, Example 1] an one-sided reversible cellular automaton with a non-SFT sofic trace was considered; we must omit the proof, but we can show that a small modification to that example gives a one-sided reversible cellular automaton with a non-sofic trace).

This list leads to some immediate questions: Is the trace of an ultimately expansive cellular automaton necessarily sofic, or an SFT? This is known to be the case for positively expansive cellular automata $([10],[16])$. Kưrka has conjectured that this would also be the case for expansive cellular automata [12, Conjecture 30]. It is clear that the left-extension property does not imply left-expansivity, as memoryless surjective CA's have the left-extension property but are not leftexpansive. However we do not know any example of a left-expansive cellular automaton that would not also have the left-extension property (for example the right-expansive CA of Proposition 6 also has the right extension property). Whether left-expansivity implies the left-extension property or not is interesting since if it does, then we have that the trace of a surjective ultimately expansive cellular automaton is sofic, and actually it is not difficult to see that it would be even an SFT.

\section{References}

1. M. Boyle: Some sofic shifts cannot commute with nonwandering shifts of finite type. Illinois Journal of Mathematics, Volume 48, Number 4 (2004) 1267-1277

2. M. Boyle, A. Maass: Expansive invertible onesided cellular automata. Journal of the Mathematical Society of Japan 52 (2000) 725-740

3. M. Boyle, A. Maass: Erratum to: [2]

4. F. Blanchard, A. Maass: Dynamical properties of expansive one-sided cellular automata. Israel Journal of Mathematics 99. (1997) 149-174

5. B. Grünbaum, G.C. Shephard.: Tilings and patterns. A Series of Books in the Mathematical Sciences. W. H. Freeman and Company, New York (1989) 
6. J. Jalonen, J. Kari: Conjugacy of One-Dimensional One-Sided Cellular Automata is Undecidable. SOFSEM 2018: Theory and Practice of Computer Science, Lecture Notes in Computer Science, vol 10706 (2018) 227-238

7. J. Kari: The nilpotency problem of one-dimensional cellular automata. SIAM Journal on Computing 21 (1992) 571-586

8. J. Kari: Rice's theorem for the limit sets of cellular automata. Theoretical Computer Science, vol. 127 Issue 2 (1994), 229 - 254

9. J. Kari, V. Lukkarila: Some Undecidable Dynamical Properties for OneDimensional Reversible Cellular Automata. Algorithmic Bioprocesses. Natural Computing Series. Springer, Berlin, Heidelberg. (2009) 639-660

10. P. Kůrka: Languages, equicontinuity and attractors in cellular automata. Ergodic Theory and Dynamical Systems. 17. (1997) 417 - 433.

11. P. Kůrka: Topological and symbolic dynamics, vol. 11. Société Mathématique de France (2003)

12. P. Kůrka: Topological dynamics of one-dimensional cellular automata. Encyclopedia of Complexity and System Sciences (R,.A.Meyers, ed.) Part 20, 9246-9268, ISBN 978-0-387-75888-6, Springer-Verlag (2009)

13. D. Lind, B. Marcus: An introduction to symbolic dynamics and coding. Cambridge University Press. (1995) ISBN 0-521-55124-2.

14. V. Lukkarila: The 4-way deterministic tiling problem is undecidable. Theoretical Computer Science 410 (2009) 1516-1533

15. A. Maruoka, M. Kimura: Condition for Injectivity of Global Maps for Tessellation Automata. Inform. Control 32(2) (1976) 158162.

16. M. Nasu: Textile systems for Endomorphisms and Automorphisms of the Shift. Volume 546 of Memoirs of the American Mathematical Society (1995)

17. M. Nasu: The Dynamics of Expansive Invertible Onesided Cellular Automata. Transactions of the American Mathematical Society Vol. 354, No. 10 (2002) 40674084

18. M. Nasu: Textile systems and one-sided resolving automorphisms and endomorphisms of the shift. Ergodic Theory and Dynamical Systems, 28(1) (2008) 167-209

19. S. Taati: Cellular automata reversible over limit set. Journal of Cellular Automata (2007) 167-177 